\section{Radio Noise from Meteors}

AN average meteor releases energy at a rate of 100 megawatts as it is disintegrated in the Earth's upper atmosphere. A portion of this energy is converted into heat and light, and it is possible that energy is also emitted in other parts of the electromagnetic spectrum, at high and low radio frequencies. Plasma resonance of the ionized trail and deceleration of electrons and ions, for example, are possible mechanisms for radio emission. Preliminary experiments conducted by Kalashnikov ${ }^{1}$ in the U.S.S.R. seemed to show that meteors produced radio noise at the lower end of the spectrum, at a frequency of about $1 \mathrm{c} . / \mathrm{s}$. His equipment consisted of a search coil, $100 \mathrm{~m}$. in diameter, that was connected to a sensitive moving-coil galvanometer. With this magnetometer a field change of $4 \times 10^{-8}$ oersted, or $4 \times 10^{-3} \gamma$, could be detected, and pulses were obtained simultaneously with the occurrence of a meteor. I have recently repeated the magnetometer experiment, and measurements have also been made at other points in the electromagnetic spectrum, so that meteor noise has been investigated at the frequencies : 1 c./s., $30 \mathrm{Mc} / \mathrm{s} ., 218 \mathrm{Mc} / \mathrm{s}$. and $475 \mathrm{Mc} . / \mathrm{s}$.

Harvard College Observatory has operated, since 1954, two Super-Schmidt camera stations at Sacramento Peak and at Mayhill, New Mexico. These cameras obtain parallactic photographs of meteors from which their height and velocity can be deduced. The magnetometer was situated at Sacramento Peak because this site was comparatively free from magnetic interference. A search coil of 10 turns and $100 \mathrm{~m}$. in diameter was laid $6 \mathrm{in}$. below the surface of the ground and was connected to a low-frequency amplifier ${ }^{2}$. On magnetically quiet days it was possible to detect a test pulse of $3 \times 10^{-3} \gamma$, a sensitivity similar to that of the Russian equipment. The signal from the magnetometer was displayed on a pen recorder. An observer at the camera site marked the record by operating a push-button to show the instant of occurrence of a meteor. The 30-Mc./s. and $475-\mathrm{Mc} / \mathrm{s}$. radiometers were situated at the Mayhill site and a similar recording procedure was followed. The 218-Mc./s. equipment placed at the Agassiz Station of Harvard College Observatory, at Harvard, Massachusetts, consisted of a Dicke-type radiometer in which the antenna signal was compared with the noise in a constant-temperature resistor. Photographs were not taken at Agassiz, but the occurrence of meteors was noted visually. The sensitivity, type of antenna and other details of each equipment are given in Table 1. The flux of 1 Jansky unit, used to define sensitivity, is equal to 1 watt $\mathrm{m}^{2}{ }^{2}$ sec. $^{-1}$. In addition, Table 1 gives the minimum power output per unit band-width that could be detected from a meteor at a range of $150 \mathrm{~km}$.

Table 1. Charactiristics of the Equipment

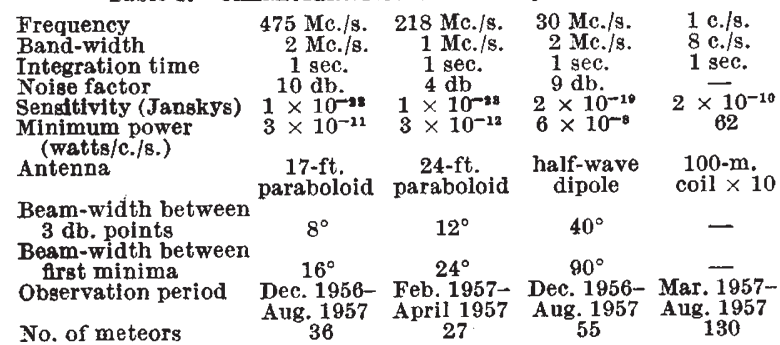

The results of the search for meteor radio noise will be given in detail elsewhere ${ }^{3,4}$. No emission from meteors was detected with any of the receiving equipments. It can be seen from Table 1 that a total of 248 meteors passed within the sensitive range of the equipment. These meteors had visual magnitudes between -1 and +5 , with a median value of $+2 \cdot 5$. Thus it is probably true to state that meteors do not emit radio noise within the frequency-range $1 \mathrm{c} . / \mathrm{s} .-500 \mathrm{Mc} / \mathrm{s}$. above the limits of sensitivity of these measurements. Meteors therefore show a surprisingly low efficiency in converting kinetic to radio energy. Their radio efficiency is certainly less than $10^{-1}$; per unit band-width. The disagreement between this work and the results of Kalashnikov is attributed to a misinterpretation of results by the latter ; a more detailed discussion will be given elsewhere ${ }^{3}$.

This research was supported jointly by the Army, Navy and Air Force under contract with the Massachusetts Institute of Technology.

Harvard College Observatory,

Gerald S. Hawrins

Cambridge, Mass., and

Boston University,

Massachusetts. April 23.

${ }^{2}$ Kalashnikov, A. G., C.R. Acad. Sci. U.S.S.R., No. 6 (1952).

2 Aarons, J., and Henissart, M., Nature, 172, 682 (1953).

- Hawkins, G. S., J. Geophys. Res. (in the press).

- Hawkins, G. S., Astrophys. J. (in the press).

\section{Radio Scintillations of Satellite 1958 $\alpha$}

THERE has been much speculation in recent years concerning the height of the irregularities in the Earth's ionosphere which are responsible for the scintillations in the signals received from discrete sources of cosmic radio emission. Hewish ${ }^{1}$ concluded that the irregularities are at a height of about $400 \mathrm{~km}$., whereas Booker ${ }^{2}$ has recently suggested that they are substantially lower-between the $E$ and $F$ regions ; other authors have found correlation between scintillation activity and the occurrence of spread- $F$ and sporadic- $E$ conditions.

Observations of the 108-Mc./s. radio signal transmitted by the artificial Earth satellite $1958 \alpha$ began in Sydney soon after its launching on February 1, and continued until March 10 . The object was, first, to discover whether the satellite's radio signal exhibited scintillations and, secondly, if such scintillations existed, to use them for obtaining a more direct estimate of the height of the electron irregularities.

Scintillations were in fact often observed, and some of the records obtained are reproduced in Fig. I. Here $a$ refers to the 108-Mc./s. signal recorded on a night when scintillation was negligible and $b$ to the signal when propagated through a highly disturbed ionosphere. The recordings $c$ and $d$ showed marked scintillations when the satellite was observed near perigee, for which the vertical height was $350 \mathrm{~km}$. These observations were made with a half-wave dipole located $\lambda / 4$ above a horizontal plane reflector and connected to a sensitive receiver of band-width $100 \mathrm{kc} / \mathrm{s}$. The output recorder had a very fast response time (less than 0.02 sec. full-scale deflexion) and ran at a chart-speed of $2.5 \mathrm{~cm}$. $/ \mathrm{sec}$.

The recordings are seen to contain two types of modulation-fast, irregular fluctuations which are identified as scintillations, and an apparently regular modulation with a period of approximately 7 sec. 